DOI: $10.5455 / 2320-1770$. ijrcog20150201

Review Article

\title{
Imaging in ovarian cancer
}

\section{Shripad S. Hebbar, Nilufer Moideen*}

Department of Obstetrics \& Gynaecology, KMC Manipal, Manipal University, Manipal, Karnataka, India

Received: 25 December 2014

Accepted: 11 January 2015

\section{*Correspondence:}

Dr. Nilufer Moideen,

E-mail: nilufermoideen@gmail.com

Copyright: (C) the author(s), publisher and licensee Medip Academy. This is an open-access article distributed under the terms of the Creative Commons Attribution Non-Commercial License, which permits unrestricted non-commercial use, distribution, and reproduction in any medium, provided the original work is properly cited.

\begin{abstract}
Ovarian cancer is the fifth most common cause of death from cancer in women after lung, breast, colon, and pancreatic cancer. The incidence of ovarian cancer rises continuously between the ages 30 and 70 years and peaks at 59 years of age. Ovarian cancer is more lethal than endometrial and cervical cancer combined due to delayed diagnosis.

Owing to the lack of symptoms and early peritoneal dissemination, the tumour has spread outside the pelvis in approximately $70 \%$ of cases at the time of diagnosis. The stage at diagnosis is the most important prognostic factor. The 5-year survival rates drop from $93 \%$ in patients with localized disease to $28 \%$ in those with distant metastases. Bimanual pelvic exam and CA 125 levels have failed to allow consistent detection of ovarian malignancy.CA 125 is a high molecular weight glycoprotein recognised by the OC 125 monoclonal antibody (sensitivity \& specificity is better with combination of TVS). Since the sensitivities of these techniques are below $50 \%$, particularly ultrasonography, $\mathrm{CT}$ and MRI has become indispensable in detection and staging of ovarian malignancy.
\end{abstract}

Keywords: Ovarian cancer, Ultrasound, Computed tomography, Magnetic resonance imaging, Risk malignancy

\section{INTRODUCTION}

Modern imaging is emerging as an important adjunct to the clinical assessment of ovarian cancer, contributing to tumour detection, characterization, staging, treatment planning and follow-up. Imaging combined with clinical findings helps to optimize patient care and assist in developing a patient-specific management plan. An imaging study should never be ordered routinely, but should be reserved for such patients where imaging results will impact on their further management.

Proper preoperative staging by imaging can prevent unnecessary surgeries in case of advanced cases of ovarian carcinoma which leads to morbidity which is preventable. Accurate staging using imaging helps to decide on neoadjuvant chemotherapy which will be ideal for patients with advanced carcinoma which helps in optimal tumour debulking during interval cytoreductive surgeries. ${ }^{1}$

\section{IMAGE CHARACTERISATION OF OVARIAN CANCER}

The role of imaging in tumor characterization has two aspects. First, in patients with a known or suspected adnexal mass, ultrasound (US) is extremely accurate in the differentiation of uterine from adnexal masses, and in the characterization of an adnexal mass as benign or malignant. ${ }^{2}$ US lesion characterization requires the use of both morphologic and Doppler findings. The greatest value of US is in the diagnosis of benign ovarian lesion. ${ }^{3}$ Ultrasound is also accurate and reliable in the characterization of ovarian cancer. However, there are a number of adnexal lesions where US findings may be equivocal or indeterminate, particularly in the diagnosis of endometrioma and cystic teratoma.

Image characterisation by $\mathrm{CT} / \mathrm{MRI}$ is classically based on internal morphology, tissue characterisation, vascularity and topographic features. MRI is recommended for the 
further evaluation of sonographically indeterminate adnexal masses. MRI should be reserved for those cases to obviate surgery in cases of sonographically indeterminate masses and result in net cost savings. Gadolinium-enhanced images are an essential component of an MRI study performed for characterization of an adnexal mass. MRI demonstrates high inter- and intraobserver agreement in the characterization of adnexal masses. ${ }^{4}$

While CT has not traditionally been used to characterize adnexal masses, the RDOG study showed CT to be equivalent to US. ${ }^{5} \mathrm{CT}$, particularly spiral $\mathrm{CT}$, can often depict the morphologic features that are used to distinguish benign and malignant masses. The second role of imaging with respect to tumor characterization is in patients with peritoneal malignancy of indeterminate etiology. In such patients, CT is extremely useful in demonstrating disease extent and the presence or absence of adnexal masses. The pattern of disease may help suggest alternative diagnoses such as primary peritoneal carcinomatosis, pseudomyxoma peritonei, metastatic disease from a non-ovarian primary malignancy, and rare peritoneal malignancies such as lymphoma or mesothelioma.

\section{THE ROLE OF IMAGING IN SURGICAL PLANNING}

The role of imaging is to aid in the evaluation of tumor location, volume and extent; this contributes to planning the surgical approach, and treatment management in patients in whom disease may be deemed nonresectable. According to a prospective study done comparing efficacy of CT and MRI in staging ovarian carcinoma, it was opined that CT should be considered the primary modality for staging ovarian cancer. MRI may be equally or more accurate than CT, but is currently limited by expense, availability, prolonged duration of scanning, and lack of widespread reader experience. ${ }^{6}$

Peritoneal metastases appear as nodular or plaque-like enhancing soft tissue masses of varying size, and may occur anywhere in the peritoneal cavity. Delayed enhancement of perihepatic implants on MRI is useful finding in improving lesion detection. Common sites of peritoneal implants are the pouch of Douglas, hepatorenal fossa, paracolic gutters, subphrenic space, and greater omentum. Lymphatic spread may occur to iliac, retroperitoneal and inguinal nodes. Superior diaphragmatic adenopathy is detected in $15 \%$ of patients with FIGO stage 3 ovarian cancer and the presence of diaphragmatic nodes is an independent negative prognostic factor. ${ }^{7}$ Hematogenous spread is a late occurrence, and parenchymal metastases in the liver or spleen are rare at initial presentation. Inoperable ovarian cancer is suggested by extensive pelvic sidewall invasion or by tumor deposits $>1-2 \mathrm{~cm}$ in the following sites; upper abdomen-along with diaphragm, porta hepatis, intersegmental fissure of the liver, lesser sac, or gastrosplenic ligament and lymph node enlargement above the renal hilum. Manifestations of stage IV disease, such as parenchymal hepatic metastases, pleural or pulmonary nodules, and superior diaphragmatic adenopathy, are important to recognize as they have grave prognostic prediction and will impact patient management.

Clinical assessment and measurement of CA-125 are the primary methods used to detect recurrent disease. Imaging can be used to confirm recurrent disease particularly when clinical assessment is suspicious or when the CA-125 level is rising. Imaging documentation of recurrent tumor can assist in the decision to perform secondary surgical cytoreduction, and may provide an objective measure for evaluation of treatment response to chemotherapy. In general, CT is preferred to MRI for imaging of recurrent disease.

Positron Emission Tomography (PET)/CT was seen superior to pelvis US, abdomino-pelvic CT, and pelvic MRI for diagnosis of malignant ovarian tumors and is useful in revealing metastatic ovarian cancer and coexisting malignant tumors. PET/CT not only helps in diagnosing recurrence but also affects subsequent management. With its ability to precisely localize the lesion as well as the extent of recurrence and distant metastases, PET/CT plays an important role in restaging for recurrent ovarian cancer and hence was suggested to be used during pre-operative evaluation of patients suspected to have ovarian cancer according to a recent study. PET/CT is limited in its ability to identify lesions $<1 \mathrm{~cm}$, in particular, those smaller than $5 \mathrm{~mm}$, leading to a false-negative rate of 5\%-10\%. Moreover, in the abdomen and pelvis, a large number of misinterpretations can occur as a result of the physiologic uptake in bowel and bladder and as a result of bowel peristalsis; knowledge of these issues and careful review are required for accurate interpretation. Similarly, in recurrent ovarian cancer, PET/CT is somewhat limited in its ability to distinguish postoperative inflammatory changes from tumour recurrence or persistence, but correlation with the $\mathrm{CT}$ findings and the patient's history and examination helps to determine the significance and thus guide management. ${ }^{8}$

\section{MORPHOLOGICAL FEATURES OF OVARIAN TUMOURS ON USG}

Ultrasonography is an easily available and accessible non-invasive invaluable diagnostic tool. Its cost is also relatively much less as compared to other imaging modalities like CT scan or MRI. Ultrasonography can therefore be of immense help in detecting and evaluating different types of ovarian tumours. Also non- neoplastic ovarian lesions requiring simple or no treatment can be differentiated from those neoplastic ones requiring prompt surgical intervention or other modalities of management. Based on the precise pre-operative judgement, exploratory laparotomy can be avoided in 
$67.2 \%$ of the ovarian pathologies which are replaced by either conservative operative laparoscopies or expectant management. ${ }^{8}$

\section{Surface epithelial tumours}

Sonographically serous cystadenocarcinomas are usually large tumours, usually presents as multilocular cystic masses and are usually bilateral. Multiple papillary projections arising from the cyst wall and multiple septae are usually encountered. Septae and walls are thick and septal thickness $>3 \mathrm{~mm}$ is suggestive of malignancy. Ascites is frequently seen.

Mucinous cystadenocarcinomas are less frequently bilateral; usually large multiloculated cystic masses containing papillary projections and echogenic material as cyst content. Penetration of tumour capsule or rupture may lead to pseudomyxoma peritoneii, which is sonographically similar to ascites or may contain multiple septations in the fluid that fills much of pelvis and abdomen.

Endometrioid tumour usually presents as a cystic mass containing papillary projections and solid areas, although in some cases predominantly solid mass that contain areas of haemorrhage or necrosis are also encountered.

Clear cell tumour sonographically presents as a nonspecific, complex, predominantly cystic mass.

Transitional tumour presents as hypoechoeic solid masses, calcification may occur in the outer wall.

\section{Germ cell tumours}

Germ cell tumours are the most common ovarian malignancies in children and young adults. When a large predominantly solid ovarian mass is present in a girl or young women, the possibility of germ cell tumour should be strongly considered.

Immature teratoma presents as a predominantly solid mass on ultrasonography, but cystic structures of varying size may also be seen. Calcifications are also commonly seen depending upon the contents of the tumour.

Dysgerminoma and yolk sac tumour are predominantly echogenic solid tumours but may contain small anechoic areas caused by haemorrhage or necrosis.

\section{Sex cord stromal tumors}

Granulosa cell tumors are predominantly unilateral. Adult granulosa cell tumors have a variable appearance, ranging from small solid masses to tumors with variable degrees of haemorrhage or fibrotic changes to multilocular cystic lesions.
Sertoli leydig cell tumor appear on are usually seen as solid hypoechoic masses on sonography and can mimic the appearance of granulosa cell tumor Thecoma and fibroma sonographically have characteristic appearance. A hypoechoic mass with marked posterior attenuation of the sound beam is seen as a result of homogenous fibrous tissue in these tumors.

\section{Metastatic tumors}

Krukenberg tumors are usually bilateral solid masses with clear well defined margins but they can become necrotic and present as complex predominantly cystic appearance that simulate primary cystadenocarcinoma on sonography. A concurrent gastric tumour detected on ultrasonography can add on to confirming the diagnosis.

Lymphoma sonographically appears as a bilateral ovarian tumour, homogenous in character and when present in the absence of ascites, aids in diagnosis. If any ovarian tumour can be detected and characterized at an earlier stage, early and proper plan of management could be decided.

Thus, ultrasonography as a diagnostic tool alone can play a very vital role in patient management and consequently indirectly in improving the prognosis of malignant ovarian tumours which otherwise would go undetected and remain untreated until very last stages. Also, screening of ovarian cancer by using ultrasound can help in early detection of ovarian cancers, so that timely management could be undertaken before the tumour has metastasized.

\section{ROLE OF MRI \& CT IN ADNEXAL LESION CHARACTERISATION AND STAGING}

Transvaginal ultrasound is the preferred technique for initial evaluation of ovarian tumour because of its availability, high resolution, and lack of ionizing radiation. A wide range of sensitivities and specificities, $85-100 \%$ and $52-100 \%$, respectively, has been reported for detection of ovarian malignancies using ultrasound. ${ }^{9}$

When used for further evaluation of an indeterminate mass seen on ultrasound in a prospective series, contrastenhanced MRI showed sensitivity and specificity of $100 \%$ and 94\%, respectively, in diagnosis of malignancy. ${ }^{9}$

\section{IMAGING FINDINGS FOR SURFACE EPITHELIAL TUMOURS}

Findings that strongly support the diagnosis of epithelial ovarian cancer include ${ }^{10}$

* Unilateral or bilateral solid cystic ovarian mass

* Multiloculated lesion with thick ( $>3 \mathrm{~mm})$, sometimes irregular enhancing septations 
* Non fibrous components

* Papillary excrescences

\section{Secondary signs}

\section{* Ascites \\ * Peritoneal implants \\ * Lymph node enlargement}

\section{Malignant germ cell tumors}

\section{Dysgerminoma}

On CT and MRI, dysgerminomas typically present as unilateral, multilobulated, well-delineated solid masses. On MRI, they exhibit low signal intensity on T1weighted images and intermediate signal on T2-weighted images. Well enhancing internal fibrovascular septations and central areas of necrosis or hemorrhage may be seen. CT may also demonstrate a solid lesion with speckled calcifications.

\section{Immature teratoma}

On imaging, immature teratomas present as unilateral, predominantly solid or mixed solid and cystic tumors, usually with a diameter greater than $10 \mathrm{~cm}$. Cystic immature teratomas may only rarely contain fluid-fat levels. Scanty or punctate areas of fat, however, and coarse calcifications in a child or young adolescent are suggestive of immature teratoma. In contrast to dermoids, immature teratomas are usually solid lesions containing cysts, and calcifications are not located murally but within the mass.

\section{Sex cord-stromal tumors}

\section{Granulosa cell tumor}

On CT and MRI, granulosa cell tumors typically present as ovarian tumors that vary considerably in size but have an average diameter of $12 \mathrm{~cm}$. They can display a broad spectrum of findings, from entirely cystic to completely solid ovarian lesions. However, the most common appearance is a predominantly sponge like, cystic, multilocular mass or a cystic and solid tumor.

\section{Sertoli-leydig cell tumor}

On imaging, Sertoli-Leydig cell tumors tend to be unilateral, enhancing solid masses of variable size. Some of these tumors may be very small and difficult to detect by imaging, although they cause hormonal effects. They may also appear as predominantly solid masses, often with peripheral cysts, or as cystic lesions with polypoid mural structures or are completely cystic.

\section{Ovarian lymphoma}

On imaging, ovarian lymphomas appear as unilateral or bilateral solid tumours. On CT they are mildly enhancing, homogeneous masses without ascites, necrosis, or calcifications. On MRI, ovarian lymphoma exhibits intermediate signal intensity on T1-weighted images and low to intermediate signal intensity on $\mathrm{T} 2$-weighted images.

\section{Metastases to the ovaries}

Approximately $5 \%$ to $15 \%$ of malignant ovarian tumors are metastasis. Stomach, colon, breast, and lung cancers are the most commonly encountered neoplasms that metastasize to the ovaries. Bilateral involvement is a typical feature, occurring in up to $75 \%$ of cases of ovarian metastasis. $40 \%$ to $50 \%$ of ovarian metastases are Krukenberg tumors, which are metastatic signet-ring cell adenocarcinomas arising most commonly from the stomach.

On imaging, two types of ovarian metastases can be differentiated. Krukenberg's tumors display characteristic imaging features, including bilateral oval, often lobulated tumors that tend to preserve the contour of the ovary. They are solid or predominantly solid with central necrosis or cysts. On MRI they display medium signal intensity on T1-weighted images and an inhomogeneous low to intermediate signal intensity on T2-weighted images. On CT and MRI they tend to show strong contrast enhancement of solid components or septations. Imaging findings in non-Krukenberg ovarian metastases may be similar to those in primary ovarian cancer, making definitive differentiation impossible

\section{STAGING BY CT AND MRI}

Staging criteria for CT and MRI have been adapted from modified FIGO staging of ovarian cancer.

Stage I ovarian cancer is confined to one (stage IA) or both (stage IB) ovaries. The capsule of the tumor should be intact, and there should be no evidence of tumor spread to the ovarian surface. In stage IC tumor is detected on the ovarian surface or the capsule has ruptured. Pelvic ascites may be present.

Stage II is described as tumor extension locally into the pelvic soft tissues and to organs within the true pelvis. In stage IIA, there is involvement of either the uterus or the fallopian tubes. Findings supporting this stage include distortion or irregularity between the interface of the tumor and the myometrium. In stage IIB, pelvic tissues such as the bladder, rectum, and pelvic peritoneum are involved. Invasion of bowel or bladder is suggested by loss of the tissue plane between the solid components of the tumor, encasement, or localized wall thickening. A distance less than $3 \mathrm{~mm}$ between the ovarian mass and the muscular pelvic sidewall or displacement or 
encasement of the iliac vessels is highly suggestive of pelvic sidewall invasion. Stage IIC ovarian cancer is defined as either stage IIA or IIB findings along with the presence of pelvic ascites.

Approximately $70 \%$ of patients present with stage III ovarian cancer, which is characterized by peritoneal implants outside the pelvis or inguinal or retroperitoneal lymphadenopathy. Large amounts of ascites are the hallmark of this stage. Typical sites of peritoneal implants include the omentum, liver surface, diaphragm, mesentery, bowel surface, and parietal peritoneum. Peritoneal dissemination is characterized by peritoneal thickening, lesions projecting from the peritoneal surfaces, or nodular lesions or stellate nodules located within the mesentery or omentum.

Stages IIIA to IIIC are advocated depending on the size of the abdominal peritoneal lesions. In stage IIIA the tumor is grossly limited to the pelvis, but large amounts of ascites indicate upper abdominal tumor spread. In stage IIIB peritoneal implants measure $2 \mathrm{~cm}$ or less whereas in stage III $\mathrm{C}$ the implant size exceeds $2 \mathrm{~cm}$. Presence of retroperitoneal and inguinal lymphadenopathy also falls under stage IIIC ovarian cancer. Stage IV ovarian cancer is characterized by distant metastases at any location outside the pelvis that have not spread peritoneally. Pleural effusion associated with pleural nodularity or focal pleural thickening suggests stage IV, but positive cytology must be obtained for final staging. It is particularly important to differentiate liver surface metastases that presents as tumour deposits with smooth margins and an elliptical or biconvex shape from liver parenchymal metastases to assign stage III or IV respectively.

\section{KEY IMAGING FEATURES (USG/CT/MRI) IN DIAGNOSIS OF OVARIAN MASSES ${ }^{11}$}

- Features suggestive of malignant epithelial tumors include a thick irregular wall, thick septa $>3 \mathrm{~mm}$, papillary projections and presence of solid areas.

- Ovarian tumors associated with endometrial hyperplasia or carcinoma include endometrioid carcinoma and granulosa cell tumor.

- Solid ovarian tumors that have very low signal intensity on T2-weighted MR images include fibroma, Brenner tumor, and, occasionally, fibrothecoma.

- The presence of fat signal intensity in an ovarian lesion especially in a young female is highly specific for a teratoma. Immature teratomas are predominantly solid with small foci of lipid material and scattered calcifications and has speckled appearance.
- Sertoli-Leydig cell tumor, struma ovarii, and cystadenofibroma presents as highly enhancing solid portions in ovarian mass.

- Calcifications are frequently encountered in serous epithelial tumor, fibrothecoma, mature or immature teratoma and Brenner tumor.

\section{ROLE OF IMAGE GUIDED STAGING}

Pre-treatment assessment by CT or MRI is helpful to identify patients who might benefit from neoadjuvant chemotherapy. Such patients may be unfit for surgery or may have an extensive tumor load that cannot be debulked. Image-guided biopsy provides a safe and rapid histologic proof of ovarian cancer in these patients, obviating the need for more invasive diagnostic procedures before the delivery of neoadjuvant chemotherapy.

Criteria for nonresectable disease may vary, but they are generally based on the size and site of implants. It includes deposits of more than $2 \mathrm{~cm}$ in the upper abdomen - along the diaphragm, porta hepatis, intersegmental fissure of the liver, lesser sac, or gastrosplenic ligament, lymph node enlargement above the renal hilum. Special sites such as abdominal wall invasion or retroperitoneal pelvic implants are also considered nonresectable. Both CT and MRI can identify these lesions equally well, and their accuracy in predicting nonresectable disease is reportedly $93 \%$ to $96 \%$. $^{12}$

Borderline tumors are rarely diagnosed preoperatively because they lack diagnostic imaging features that distinguish them from benign or early malignant epithelial tumors. On MRI, borderline tumors are predominantly cystic, with fluid ranging in $\mathrm{T} 1$ and $\mathrm{T} 2$ signal because of varying concentrations of protein and mucin. On CT and MRI, borderline tumors tend to be large unilateral or bilateral, predominantly cystic ovarian tumors that cannot be confidently distinguished from invasive ovarian cancers. Papillary projections measuring 10 to $15 \mathrm{~mm}$ are more frequently found in borderline tumors than in benign and malignant epithelial ovarian tumors.

\section{THE ROLE OF RMI (RISK OF MALIGNANCY INDEX) SCORING SYSTEM IN DETECTING OVARIAN MALIGNANCY}

The RMI scoring system is the method of choice for predicting whether or not an ovarian mass is likely to be malignant. This scoring system calculates scores using ultrasound features, menopausal status and preoperative CA125 level according to the equation:

RMI score $=$ ultrasound score $\mathrm{x}$ menopausal score $\mathrm{x}$ CA125 level in $\mathrm{U} / \mathrm{ml}$. 
Ultrasound features: Multilocular cyst, Solid areas, bilateral lesions, ascites, intra-abdominal metastases.

The RMI, now termed RMI 1, was developed in the 1990s by Jacobs et al. Tingulstad et al. developed their version of the RMI in 1996, which is now known as RMI 2. In 1999, Tingulstad et al. modified the RMI, termed RMI 3. Yamamoto et al., who created their own model of a malignancy risk index, added the parameter of tumor size to the RMI, and have termed it RMI $4 .{ }^{13}$

Differences between different RMI scoring systems are explained below:

RMI $1=\mathrm{U} \times \mathrm{M} \times \mathrm{CA}-125$, where a total ultrasound score of 0 made $U=0$, a score of 1 made $U=1$, and a score of $\geq 2$ made $U=3$; premenopausal status made $\mathrm{M}=1$ and postmenopausal $M=3$. The serum level of $\mathrm{CA}-125$ was applied directly to the calculation.

RMI $2=\mathrm{U} \times \mathrm{M} \times \mathrm{CA}-125$, where a total ultrasound score of 0 or 1 made $U=1$, and a score of $\geq 2$ made $U=4$; premenopausal status made $\mathrm{M}=1$ and postmenopausal $\mathrm{M}=4$. The serum level of CA-125 was applied directly to the calculation.

RMI $3=\mathrm{U} \times \mathrm{M} \times \mathrm{CA}-125$, where a total ultrasound score of 0 or 1 made $U=1$, and a score of $\geq 2$ made $U=3$; premenopausal status made $\mathrm{M}=1$ and postmenopausal $\mathrm{M}=3$. The serum level of CA-125 was applied directly to the calculation.

RMI $4=\mathrm{U} \times \mathrm{M} \times \mathrm{S}$ (size in centimetres) $\times \mathrm{CA}-125$, where a total ultrasound score of 0 or 1 made $U=1$, and a score of $\geq 2$ made $U=4$. Premenopausal status made $M=1$ and postmenopausal status made $\mathrm{M}=4$. A tumor size (single greatest diameter) of $<7 \mathrm{~cm}$ made $S=1$, and $\geq 7 \mathrm{~cm}$ made $\mathrm{S}=2$. The serum level of CA-125 was applied directly to the calculation.

\section{WOMEN WITH AN RMI SCORE >200 IS PREDICTIVE OF OVARIAN MALIGNANCY}

The malignancy risk index is more accurate than the menopausal status, serum CA 125 levels, and ultrasound features separately in diagnosing malignancy. According to our institutional study, no statistically significant difference in the performance of three different malignancy risk indices (RMI 1, RMI 2, RMI 3, RMI4) in identifying malignancy. ${ }^{13}$

The use of RMI scoring is not appropriate when obvious metastatic disease has been identified by ultrasound. In this situation a CT/MRI scan is to be obtained for more information on the extent of metastatic disease. CT is better than US for retroperitoneal assessment, and the detection of omental and peritoneal disease.

It is impossible to differentiate subtypes of ovarian cancer by imaging, but serous cancers are commonly bilateral and may contain calcifications on CT. Endometrioid cancer is commonly solid.

\section{COMPARISON OF FOUR RMI SCORES}

Table 1: Comparison of four RMI Scores.

\begin{tabular}{|c|c|c|c|c|}
\hline RMI & $\mathbf{U}$ & $\mathbf{M}$ & CA 125 & $\begin{array}{l}\text { Tumor } \\
\text { size }\end{array}$ \\
\hline RMI 1 & $\begin{array}{l}0=0 \\
1=1 \\
\geq 2=3\end{array}$ & $\begin{array}{l}\text { Premenopausal }=1 \\
\text { Postmenopausal }=3\end{array}$ & $\mathrm{U} / \mathrm{ml}$ & - \\
\hline RMI 2 & $\begin{array}{l}0 / 1=1 \\
\geq 2=4\end{array}$ & $\begin{array}{l}\text { Premenopausal=1 } \\
\text { Postmenopausal }=4\end{array}$ & $\mathrm{U} / \mathrm{ml}$ & \\
\hline RMI 3 & $\begin{array}{l}0 / 1=1 \\
\geq 2=3\end{array}$ & $\begin{array}{l}\text { Premenopausal }=1 \\
\text { Postmenopausal }=3\end{array}$ & $\mathrm{U} / \mathrm{ml}$ & \\
\hline RMI 4 & $\begin{array}{l}0 / 1=1 \\
\geq 2=4\end{array}$ & $\begin{array}{l}\text { Premenopausal }=1 \\
\text { Postmenopausal }=4\end{array}$ & $\mathrm{U} / \mathrm{ml}$ & $\begin{array}{l}<7 \mathrm{~cm}=1 \\
\geq 7 \mathrm{~cm}=2\end{array}$ \\
\hline
\end{tabular}

\section{COMPARATIVE STUDIES ON USG, CT/MRI CORRELATED WITH SURGICO- PATHOLOGICAL FINDINGS}

There are two major diagnostic challenges when an ovarian mass is detected: the determination of malignancy and the evaluation of tumour extent (staging). Diagnostic studies that allow accurate confirmation of benignity might reduce unnecessary surgery. Diagnostic studies that allow accurate cancer staging should help determine surgical and chemotherapeutic planning.

Johnson RJ (1993) reviewed the role of radiological imaging in the management of ovarian cancer and has opined that $\mathrm{CT}$ is the single best and most practical approach in staging ovarian cancer. The staging accuracy of CT is $70 \%$ to $90 \%{ }^{14}$

Ultrasound (US) performed with transabdominal and endovaginal techniques has demonstrated accuracies of up to $80 \%$ in evaluation of ovarian masses; US is better in characterisation of masses than in staging of malignancy. Studies of contrast material - enhanced CT and MR imaging have shown accuracies of almost $80 \%$ in diagnosis of cancer and $80 \%-90 \%$ in detection of abdominal spread. ${ }^{15}$ Cross-sectional imaging can provide staging information which can assist in surgical planning and in selection of treatment options. The choice of imaging modality depends on the information required, equipment available, local expertise, and preference. Ultrasound plays a pivotal role in ovarian tumour detection and characterization but its use in staging ovarian cancer is limited.

Only two studies have compared all three of these modalities in diagnosis of ovarian cancer and its spread Kurtz AK et al (1999) in The Radiology Diagnostic Oncology Group (RDOG) performed a 3 year, 
prospective study of nearly 300 patients with ovarian masses. ${ }^{14}$ They evaluated the results of Doppler and conventional ultrasound, CT and MR imaging and compared their performances in diagnosis of malignant ovarian mass and staging and its spread. They proved that all three imaging modalities had high accuracy of almost $91 \%$ in diagnosing malignancy. ${ }^{14}$ In the ovaries, accuracy of MR imaging (91\%) was significantly greater than other two modalities. In diagnosing tumour dissemination to pelvis and the abdomen, conventional US, CT, and MR imaging had similar accuracies (0.87-0.95). In differentiation of disease confined to the pelvis from abdominal spread, the specificity of conventional US (96\%) was higher than that of CT and significantly higher than that of MR imaging (88\%), whereas the sensitivities of MR imaging (98\%) and CT (92\%) were significantly higher than that of conventional US (75\%). They concluded that there is only little variation among conventional ultrasound, CT and MR imaging in staging ovarian cancer, however MR imaging may have a substantial role in the diagnosis of peritoneal disease and was superior in diagnosis of malignant ovarian masses.

Rieber A et al. (2001) has compared the role of MRI with Transvaginal sonography (TVS) and PET scans in adnexal masses and stated that TVS is the diagnostic method of choice as a screening technique for ovarian masses. ${ }^{15}$ Suspicious findings on transvaginal sonography should be confirmed on MR imaging before undertaking any surgical procedures. MR imaging, transvaginal sonography, PET has sensitivities, 83\%, 92\%, 58\% specificities, 84\%, 59\%, 78\%; diagnostic accuracies, $83 \%, 63 \%, 76 \%$, respectively in diagnosing ovarian masses. $^{15}$

Hricak H (2001) in the study on imaging of ovarian cancer has stated that Ultrasound coupled with Doppler is accurate for characterisation of adnexal masses whereas $\mathrm{CT}$ imaging is suggested for staging of primary ovarian cancer. $^{16}$

CT has been suggested as the imaging modality of choice for staging ovarian cancer Javitt MC et al. (2005) in a study on staging and follow up of ovarian cancer concluded that multidetector $\mathrm{CT}$ provides all of the clinically relevant information, including size and location of peritoneal implants, lymph node enlargement, and prediction of resectability. ${ }^{17}$ The role of MRI is only in patients with contraindications to CT (e.g., use of contrast agents, pregnancy) and as in case of inconclusive CT findings. ${ }^{11}$ The accuracy of staging for ovarian cancer is $53-92 \%$ for CT and $78-88 \%$ for MRI. Spencer JA (2005) in his issue on approach to ovarian cancer diagnosis has suggested ultrasound as the first radiological investigation in a patient with suspected ovarian malignancy and CT as the investigation of choice in preoperative evaluation. MRI is reserved for only those cases with indeterminate diagnosis. ${ }^{18}$
Kim CK et al. (2009) have done a comparative study of MRI and integrated PET/CT for the preoperative detection of peritoneal carcinomatosis arising from primary ovarian cancer in 253 patients and has concluded that patient- based sensitivity, specificity and accuracy of the use of MRI versus PET/CT for the detection of peritoneal carcinomatosis were $95 \%$ versus $84 \%, 50 \%$ versus $50 \%$ and $87 \%$ versus $78 \%$ respectively. ${ }^{19}$ Moreover, the comparison of the lesion-based sensitivity, specificity and accuracy of MRI versus integrated PET/CT were $86 \%$ and $75 \%, 76 \%$ and $84 \%$, and $82 \%$ and $78 \%$, respectively concluding that MRI was more sensitive that PET/CT for detection of peritoneal carcinomatosis.

Iyer VR and Lee SI (2010), in a review article describing the role of different imaging modalities for ovarian cancer detection and adnexal lesion characterisation have concluded that CT is the preferred technique in the pretreatment evaluation of ovarian cancer, to define the extent of the disease and to assess the likelihood of optimal cytoreduction. ${ }^{9}$

Only one study has compared the efficiency of all the three imaging modalities in the staging of ovarian cancer, the study was conducted by the RDOG. CT and MR imaging are more accurate than ultrasound (US), particularly for lesions in the in the subdiaphragm and along the hepatic surface. However, the accuracy of US was also high; US can be used to supplement CT or MR imaging, especially in the hepatic substance and in the lymph nodes. Hossain F et al. (2010) have recommended the combined use of CA125 with Resistance index with colour Doppler in preoperative diagnosis of malignant ovarian tumour. ${ }^{20}$

Mubarak F et al. (2011) have found that multidetector CT have $97 \%$ sensitivity, $91 \%$ specificity, and an accuracy of $96 \%$ in the differentiation of benign and malignant ovarian masses, while PPV and NPV were $97 \%$ and $91 \%$, respectively. ${ }^{21}$ SH Chandrashekara et al. (2011) has studied the role of $\mathrm{CT}$ on diagnosis of peritoneal metastasis in ovarian malignancies observed a high specificity (94.3\%) and PPV (75.2\%) for the diagnosis of peritoneal metastases. ${ }^{22}$

According to American College of Radiology (ACR) Appropriateness Criteria reviewed in 2012 for Staging and Follow-up of Ovarian Cancer in pre-treatment staging of ovarian cancer as well as to rule out recurrent ovarian cancer, CT abdomen and pelvis with contrast again holds the highest ranking followed by MRI with or without contrast.

\section{CONCLUSION}

The present review highlights remarkable diagnostic abilities of two different imaging modalities in detection of extent of malignant spread both individually and in combination. Addition of the third parameter (CA-125) 
further improved the precision. It is difficult to suggest a single investigative modality for evaluation of women with suspected ovarian malignancy. All the three modes, though not inferior by themselves, are complimentary to each other in their diagnostic performances.

Pre- operative sonography by both routes should be performed, transvaginal ultrasound provides opportunity for characterisation of ovarian mass, transabdominal ultrasound imaging assesses extra pelvic dissemination. CT imaging augments ultrasound findings because of its ability to image the deeper areas and the minimal extra ovarian spread and proves to be important adjunct to assess and treat when surgical staging is not feasible.

Funding: No funding sources

Conflict of interest: None declared

Ethical approval: Not required

\section{REFERENCES}

1. Woodward PF, Hosseinzadeh K, Saenger FS, Radiologic staging of ovarian carcinoma with pathologic correlation. Radiographics. 2004;24:22546.

2. Hedvig Hricak, Fergus Coakley. Multidisciplinary symposium - gynaecological malignancy: imaging ovarian cancer. Cancer Imaging. 2001;2:67-70.

3. Kinkel K, Hricak H, Lu Y, Tsuda K, Filly RA. Ultrasound characterization of ovarian masses: a meta-analysis. Radiology. 2000;217:803-11.

4. Hricak H, Chen M, Coakley FV, Kinkel K, Yu KK, Sica G, et al. Complex adnexal masses: Detection and characterization with MR imaging: multivariate analysis. Radiology. 2000;214:39-46.

5. Kurtz AB, Tsimikas JV, Tempany CM, Hamper UM, Arger PH, Bree RL, et al. Diagnosis and staging of ovarian cancer: comparative values of Doppler and conventional US, CT, and MR imaging correlated with surgery and histopathologic analysis: report of the radiology diagnostic oncology group. Radiology. 1999;212:19-27.

6. Forster R, Hricak H, Occhipinti KA, Powel CB, Frankel SD, Stern JL. Ovarian cancer: staging with CT and MR imaging. Radiology. 1995;197:617-26.

7. Nelson BE, Rosenfeild AT, Schwartz PE. Preoperative abdominopelvic computed tomographic prediction of optimal cytoreduction in epithelial ovarian carcinoma. J Clin Oncol. 1993;11:166-72.

8. Nam EJ, Yun MJ, Oh YT, Kim JW, Kim JH, Kim S, et al. Diagnosis and staging of primary ovarian cancer: Correlation between PET/CT, Doppler US, and CT or MRI. Gynecol Oncol. 2010;3:389-94.

9. Veena R. Iyer, Susanna I. Lee. MRI, CT, and PET/CT for ovarian cancer detection and adnexal lesion characterization. AJR Am J Roentgenol. 2010;194(2):311-21.
10. Kinkel K, Lu Y, Mehdizade A, Pelte MF, Hricak H. Indeterminate ovarian mass at US: incremental value of second imaging test for characterization: metaanalysis and Bayesian analysis. Radiology. 2005;236:85-94.

11. Forstner R. Radiological staging of ovarian cancer: Imaging findings and contribution of $\mathrm{CT}$ and MRI. Eur Radiol. 2007;17:3223-35.

12. Haaga JR. Nonresectable disease. In: Haaga JR, eds. CT and MRI of the Whole Body. 5th ed. US: Mosby/Elsevier; 2009: 28-65.

13. Manjunath, Pratapkumar, Sujatha K, Vani R. Comparison of three risks of malignancy indices in evaluation of pelvic masses. Gynaecol Oncol. 2001;81(2):225-9.

14. Johnson RJ. Review radiology in the management of ovarian cancer. Clin Radiol. 1993;48:75-82.

15. Rieber A, Nüssle K, Stöhr I, Grab D, Fenchel S, Kreienberg R, et al. Preoperative diagnosis of ovarian tumors with MR imaging: comparison with transvaginal sonography, positron emission tomography, and histologic findings. AJR Am J Roentgenol. 2001;177:123-9.

16. Hedvig Hricak, Fergus Coakley. Imaging ovarian cancer; multidisciplinary symposium gynaecological malignancy. Digital Object Identifier. 2004; 17:456-60.

17. Javitt MC, Fleischer AC, Andreotti RF, Bohm-Velez M, Horrow MM, Hricak H, et al. Staging and follow up of ovarian cancer. Am Coll Radiol. 2005;12(3):23-6.

18. Spencer JA. A multidisciplinary approach to ovarian cancer at diagnosis. Br J Radiol. 2005;78:S94-102.

19. Kim CK, Kwan B, Choi JY, Kim JH. Comparison of the MRI and integrated PET/CT findings in the preoperative detection of peritoneal carcinomatosis arising from primary ovarian cancer. J Korean Soc Radiol. 2009;60:117-26.

20. Hossain F, Karim MN, Rahman SM, Khan N, Siddiqui M, Hussain R. Preoperative detection of ovarian cancer by color Doppler ultrasonography and CA 125. Bangladesh Med Res Counc Bull. 2010;36:68-73.

21. Mubarak F, Alam MS, Akhtar W, Hafeez S, Nizamuddin N. Role of multidetector computed tomography (MDCT) in patients with ovarian masses. Int J Women's Health. 2011;3:123-6.

22. Chandrashekara SH, Thulkar S, Srivastava DN, Kumar L, Hariprasad R, Kumar S, et al. Preoperative evaluation of peritoneal deposits using multidetector computed tomography in ovarian cancer. Br J Radiol. 2011;84:38-43.

DOI: $10.5455 / 2320-1770$. ijrcog20150201

Cite this article as: Hebbar SS, Moideen N. Imaging in ovarian cancer. Int J Reprod Contracept Obstet Gynecol 2015;4:1-8. 